\title{
Treatment of spontaneous intracranial hypotension: experiences in a UK regional neurosciences Centre
}

\author{
Authors: Joanna E Perthen, ${ }^{A}$ Paul J Dorman, ${ }^{B}$ Danny Morland, ${ }^{C}$ Nancy Redfern ${ }^{C}$ and David JA Butteriss ${ }^{A}$
}

A robust treatment paradigm for spontaneous intracranial hypotension has yet to be agreed upon. We present retrospective data from the patient cohort at our UK regional neurosciences centre from 2010-2020 and describe our locally developed treatment pathway.

Seventy-three patients were identified: 31 men and 42 women; mean age was 42 years. The majority presented with a headache of variable duration, and most had positive imaging. Very few patients $(7 \%)$ responded to conservative treatment. Sixty-six underwent epidural blood patching, with 39 (59\%) having a good response. Twenty-three patients underwent myelography and targeted treatment (injection of fibrin sealant at the leak site), with 13 (57\%) showing a good response. One patient had successful surgery. The relapse rate after response to epidural blood patching was $10 \%$, and after response to targeted treatment was $23 \%$. Most patients who relapsed responded to repeated treatments.

The outcome data for our diverse patient cohort shows the success of a staged approach to treatment. Relapse rates are low, and surgery is only rarely required. We use these data to inform our discussions with patients, and present them here to enable other centres to develop robust investigation and treatment paradigms of their own.

KEYWORDS: spontaneous intracranial hypotension, CSF leak, epidural blood patch, myelography, postural headache

DOI: 10.7861/clinmed.2020-0791

\section{Introduction}

Spontaneous intracranial hypotension (SIH) is an increasingly recognised cause of headache. Patients are typically adults of working age, and although postural headache is the commonest presenting complaint, non-postural headache, cranial nerve palsies, and cognitive decline are also recognised features. ${ }^{1,2}$ Symptoms can be debilitating. Annual prevalence has been

Authors: A consultant neuroradiologist, The Newcastle upon Tyne Hospitals NHS Foundation Trust, Newcastle upon Tyne, UK; ${ }^{B}$ consultant neurologist, The Newcastle upon Tyne Hospitals NHS Foundation Trust, Newcastle upon Tyne, UK; ${ }^{C}$ consultant anaesthetist, The Newcastle upon Tyne Hospitals NHS Foundation Trust, Newcastle upon Tyne, UK estimated at about 5 per 100,000 and, although diagnostic criteria are becoming established, optimal treatment protocols remain unclear. ${ }^{3}$

$\mathrm{SIH}$ is defined by a low cerebrospinal fluid (CSF) volume within the central nervous system. This is due to a spontaneous leak of CSF into the extra-dural space within the spine, typically due to one of three mechanisms: dural tear, meningeal diverticulum or CSF-venous fistula. ${ }^{4}$ This low CSF volume leads to various compensatory physiological changes in the brain and spine in order to maintain total central nervous system (CNS) volume, including subdural effusions and venous engorgement. ${ }^{5}$ The low CSF volume leads to loss of buoyancy in the cranium and downward slumping of the midbrain and cerebellum. This downward traction on the meninges is thought to be the basis of the most common clinical symptom, a postural headache that is relieved on lying flat. In fact, both clinical features and imaging findings can vary significantly between patients depending on how long the leak has been present, the site and size of the leak, and the individual's physiology and anatomy (presumably reflecting their ability to compensate). ${ }^{1,6-8}$ Ultimately the diagnosis is made based upon a combination of clinical symptoms and radiological findings (Table 1 ).

Treatment protocols vary between institutions, with some published examples. ${ }^{9}$ The role for various interventions is now becoming fairly well accepted. An initial period of conservative management (with bed rest and increased caffeine intake) can be trialled and, if unsuccessful, epidural blood patch (EBP) can be undertaken. EBP is the injection of autologous blood into the lumbar epidural space. These unguided or 'blind' injections are known to be an effective treatment for low-pressure headache after accidental dural puncture following epidural insertion in the obstetric population, and have also been shown to be effective in $\mathrm{SIH}^{10}{ }^{10}$ Complex radiological investigations and treatments for spinal CSF leaks are also emerging, including the use of dynamic myelography to locate the leak site, with subsequent targeted injection of fibrin sealant and/or blood under imaging guidance at the leak site. There is also a role for surgery to seal refractory leaks. Previous studies have focused primarily on better understanding the underlying pathophysiology of SIH as well as presenting patient outcome data, typically using retrospective patient cohorts and assessing the response to a specific treatment. ${ }^{11}$

Here, we present retrospective data from the entire cohort of SIH patients at our regional neurosciences centre over the last 10 years. Our experience with this cohort has led to the development of a local treatment pathway, in collaboration with our colleagues 
Table 1. Summary of the clinical presentations and imaging findings of spontaneous intracranial hypotension

\section{Clinical presentations}

Headache (core symptom, patterns may vary)

Postural headache: worse with upright posture, relieved on lying down

Sudden onset severe headache causing emergency presentation New daily persistent headache

Exertional headache

\section{Associated symptoms}

Posterior neck pain/stiffness

Shoulder stiffness

Nausea and vomiting

Change in hearing / new tinnitus

\section{Unusual clinical presentations}

Cognitive (frontotemporal) decline

Cranial nerve dysfunction

Reduced consciousness level

\section{Imaging findings}

Magnetic resonance imaging of the brain

Subdural collections: haematoma or hygroma, often bilateral Prominence/dilatation of the venous sinuses

Engorgement of the pituitary gland

Downward slumping of the midbrain, flattening of the pons Cerebellar tonsillar descent

Medial and inferior migration of the medial temporal lobes Diffuse smooth meningeal thickening and enhancement Normal appearances do not exclude spontaneous intracranial hypotension

\section{Magnetic resonance imaging of the spine}

Epidural fluid collection: often longitudinally extensive Epidural venous distention

May be entirely normal in neuroradiology, neurology, anaesthetics and neurosurgery, described later. We present treatment response data for the entire cohort, to give a representative overview of this diverse patient group and enable other centres to develop robust investigation and treatment paradigms of their own.

\section{Materials and methods}

Patients diagnosed with SIH who underwent imaging and/ or treatment at our neurosciences centre between 2010 and 2020 were retrospectively identified, using neuroradiology and anaesthetic departmental records. The diagnosis of SIH was made based on the clinical symptoms and imaging findings. For inclusion in the patient cohort presented here, patients were required to have a new persistent postural headache, and/or positive imaging findings. Patients with a known cause of CSF leak within the month prior to presentation were excluded; this included lumbar puncture, significant trauma and surgery.
Each patient's demographics, presenting symptoms, imaging findings, treatments and responses were collated via a review of our local picture archiving and communication system (PACS), clinic letters and anaesthetic records. Patients were followed up clinically, and response to a specific treatment is defined as resolution of clinical symptoms or sufficient improvement in symptoms such that no further treatment is required, for a period of at least 6 months. A relapse is defined as recurrence of clinical symptoms after a period of at least 6 months of response. Radiological follow-up is typically not performed, unless there is an unexpected change in symptoms.

Our investigation and treatment pathway, developed over the last 10 years, is shown in Fig 1. We aim to perform magnetic resonance imaging (MRI) of the brain (including post-contrast imaging) and whole spine to help make the diagnosis and exclude other causes of the patient's symptoms. Spinal imaging will sometimes reveal an epidural fluid collection, though these epidural collections are often extensive and, in our experience,
Fig 1. Locally developed treatment pathway for spontaneous intracranial hypotension patients. Treatments are offered as described until a response is achieved. CT = computed tomography; CSF = cerebrospinal fluid; EBP = epidural blood patch; MRI = magnetic resonance imaging; $\mathrm{SIH}=$ spontaneous intracranial hypotension.

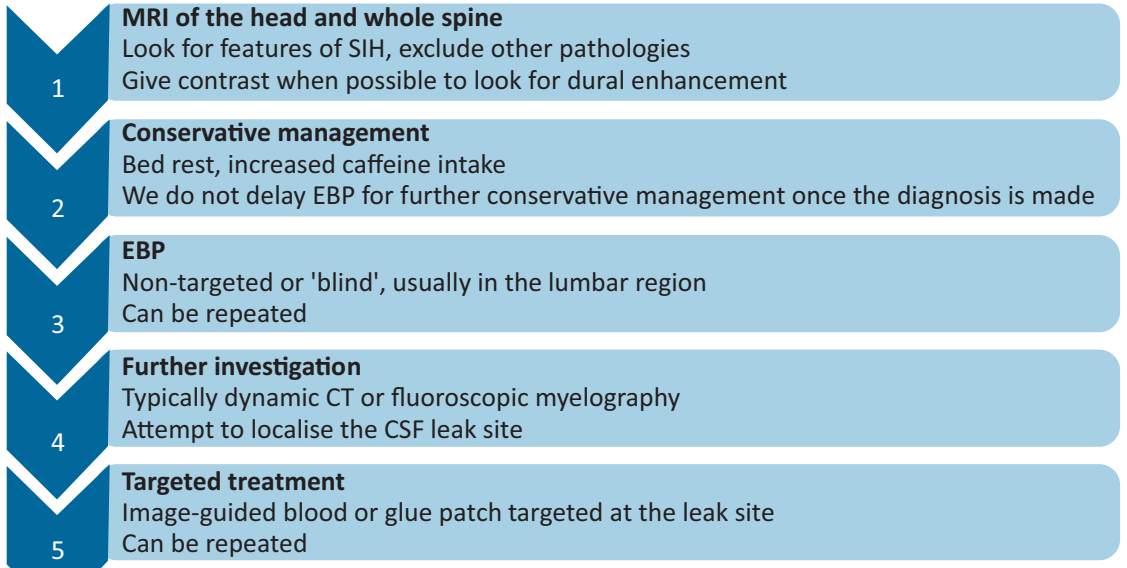

Surgery

Find and seal leak 
do not usually help to localise the leak site. By the time patients present and undergo imaging studies, they may already have tried a period of bed rest, and given that conservative treatments have a low response rate in our experience, we typically do not delay EBP once a firm diagnosis is made. In our institution, initial EBP is performed in the lumbar region by an obstetric anaesthetist, and we aim to inject at least $30 \mathrm{~mL}$. If the first blood patch produces only transient improvement, a second blood patch is offered.

Patients who do not respond to EBP are offered further investigation to attempt to localise the leak site. A variety of myelographic techniques are available, all requiring thecal puncture using a 22 gauge spinal needle. This has not been found to worsen the patient's symptoms, presumably due to the pre-existing low CSF pressure reducing the pressure gradients, even in cases of significant brain shift at the foramen magnum. Conventional computed tomography (CT) and fluoroscopic myelography, with a delay between instilling contrast and imaging, can obscure the leak site, either due to extensive opacification of extra-thecal CSF collections or due to false-localising signs (extrathecal CSF leakage, usually in the upper cervical or cervicothoracic region that does not correspond to the leak site). ${ }^{12,13}$ We use variations of dynamic CT and fluoroscopic myelography to minimise the time between instillation of contrast and imaging, with delayed imaging if no leak is identified on the initial scan. Supine, decubitus and prone Trendelenburg techniques can be used. A leak site may be identified as local extrathecal contrast, or a CSF-venous fistula with opacification of a local draining vein. Exact leak sites may be difficult to identify in very fast or very slow leaks and, in these cases, likely leak sites can be extrapolated by the presence of a density gradient of CSF opacification within the extrathecal contrast or the presence of a likely cause such as a transdural bony spicule. The leak can then be targeted with an imaging guided injection of $2-4 \mathrm{~mL}$ of fibrin sealant (Tisseel (Baxter, Deerfield, USA)), which may be combined with autologous blood for difficult to access locations or where larger volumes are desired. In our institution, we perform these targeted injections under CT guidance, though other institutions use fluoroscopy.

Although most of our patients follow the pathway described, we note that several have not, in particular, some of those who presented with atypical symptoms and/or atypical imaging and some who have expressed a specific treatment preference after discussion with the neurologist or neurosurgeon in charge of their care.

\section{Results}

Seventy-three patients with a diagnosis of SIH were retrospectively identified. The cohort consisted of 31 men and 42 women, mean age at presentation was 42 years; age range 19-76 years.

The majority of the patients (71/73) presented with a headache of variable duration, usually postural and often described as sudden onset. The headache was occasionally accompanied by other symptoms such as neck pain, cranial nerve palsy and cognitive problems. For two patients, headache was not the primary complaint: one patient presented with progressive cognitive decline and one patient with neck pain and episodes of altered consciousness.

Most of the patients (70/73) had positive imaging findings in the brain and/or spine. Of the three patients without positive findings, two had normal MRI and one had a normal CT of the head (no MRI performed for this patient prior to EBP).
The responses to treatment for the entire cohort are presented in a flow chart (Fig 2).

Only a small minority of patients $(5 / 73 ; 7 \%)$ responded to an initial trial of conservative treatment, and did not require any further intervention.

The majority (66) of the remaining 68 patients underwent EBP; $39 / 66(59 \%)$ had a good response, the majority requiring only one $(20 / 39 ; 51 \%)$ or two $(15 / 39 ; 38 \%)$ blood patches. Of the 39 responders to $\mathrm{EBP}$, four $(10 \%)$ went on to relapse at a later date, with two responding to repeat $\mathrm{EBP}$, one patient still awaiting repeat $E B P$ at the time of writing and one patient not responding to repeat EBP. This patient went on to have myelography and targeted treatment, still with no response, and is now considering surgery.

Twenty-seven of the 66 patients ( $41 \%$ ) undergoing EBP did not respond. Of these, 21 went on to have myelography and targeted treatments. Two patients proceeded directly to myelography and targeted treatment without first undergoing EBP. The first of these did not have typical imaging features of $\mathrm{SIH}$, and the second had a tethered cord and proceeding to myelography was felt to be safer than blind EBP. In total, 23 patients underwent myelography and targeted treatment. Myelography identified a definite or possible leak site for 21 of the 23 patients, and image-guided injections of fibrin sealant + - - autologous blood were performed at the suspected leak site. The two patients in whom no leak site was identified underwent bilateral patching of sequential spinal levels. One of the patients has responded to this; the other has not yet shown a response and, at the time of writing, is awaiting further treatment. Thirteen of the 23 patients (57\%) receiving targeted treatment have responded. Three of these responders subsequently relapsed and all three then responded to a further targeted treatment at the same spinal location. Nine patients remain in whom a possible leak site was identified but who did not respond to targeted treatment. Of these, six are awaiting further targeted treatments, one underwent successful surgery, and two recovered spontaneously more than 6 months after their previous treatment.

There are six patients who did not respond to EBP but have not gone on to myelography and targeted treatment ('Other' category in Fig 2). Two of these are awaiting myelography, one was too unwell for further treatment due to significant comorbidities, one underwent alternative treatment (occipital nerve stimulator), one was lost to follow-up and one recovered with a further period of conservative treatment (specifically, 1 month of bed rest).

Although treatment responses are assessed clinically rather than radiologically, some patients underwent repeat imaging after clinical improvement, sometimes for unrelated reasons, and we find that normalisation of the imaging appearances mirrors clinical response (Fig 3).

\section{Discussion}

$\mathrm{SIH}$ is a relatively recently described condition that can cause debilitating symptoms. Despite its increasing recognition and better understanding of the underlying pathophysiology, SIH probably still remains under-diagnosed. Diagnosis is essential since effective treatment options are available, though a robust treatment paradigm has yet to be agreed. We have identified 73 $\mathrm{SIH}$ patients treated at our regional neurosciences centre over the last 10 years. The majority are aged between 25 and 60 years, 
Fig 2. Patient outcome flowchart. Response refers to clinical recovery or improvement such that no further treat ment is needed for a period of at least 6 months. Relapse refers to recurrence of the original symptoms after at least 6 months improvement. Outcomes for the group labelled 'Other' are detailed in the main text. EBP = epidural blood patch.

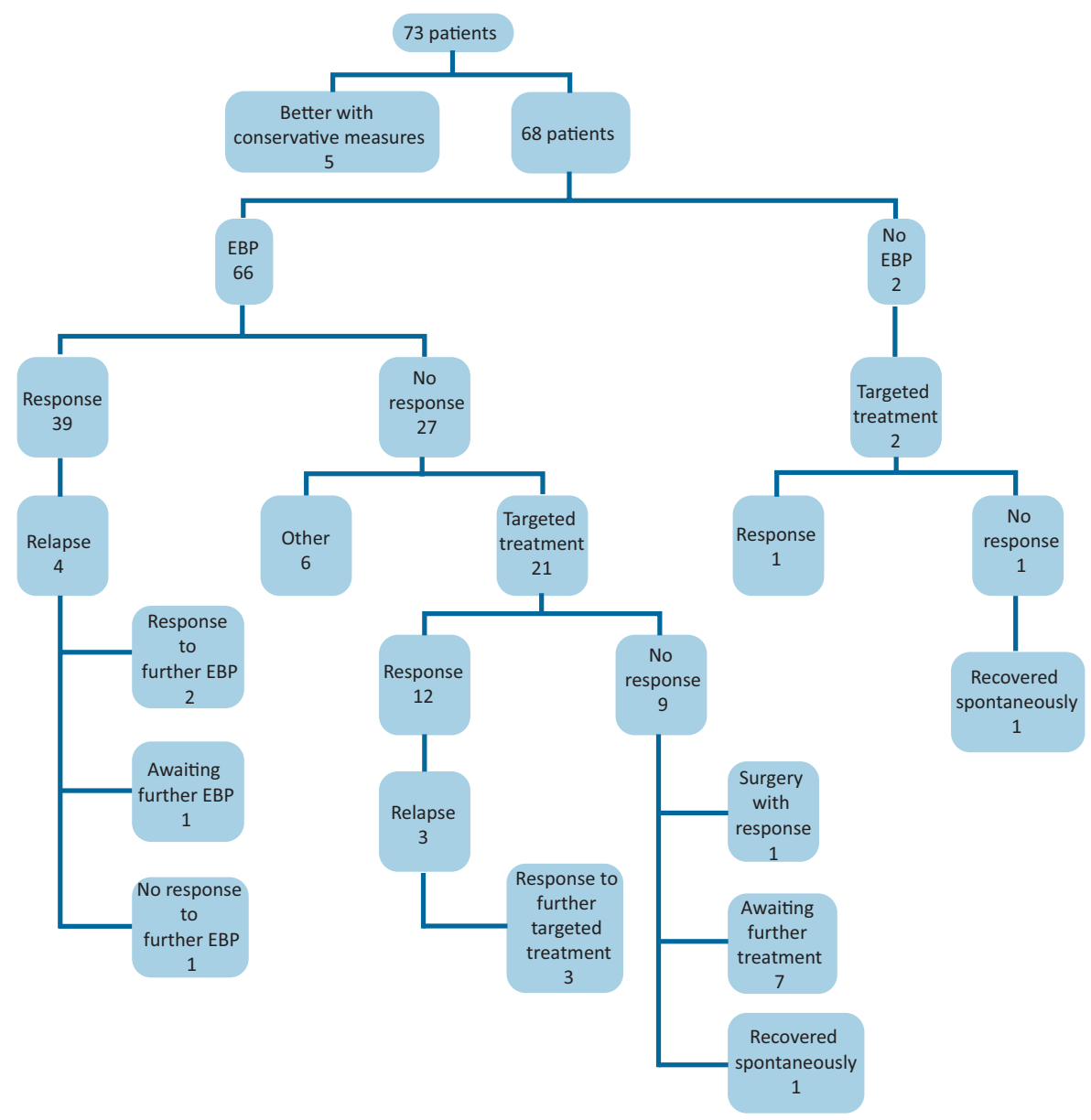

with a female preponderance. While the majority have positive imaging findings, and most complain of postural headache, some do not, highlighting the importance of considering this diagnosis even in those patients with atypical presentations and/or normal imaging.

Within our cohort, relatively few patients responded to an initial period of conservative treatment $(5 / 73 ; 7 \%)$, though three patients did subsequently spontaneously recover at some point later in the treatment pathway. Over half of those undergoing non-targeted EBP had a good response (39/66; 59\%), and we have found lumbar EBPs can be effective even when the suspected CSF leak is distant to the lumbar region. Of those undergoing myelography and targeted treatment, a definite or possible leak site was identified in the majority, and more than half had a
Fig 3. Magnetic resonance imaging of the brain. a) Imaging at presentation showing marked brainstem slumping, venous and pituitary engorgement, and cerebellar tonsillar descent. b) Imaging normalised after responding clinically to targeted treatment.
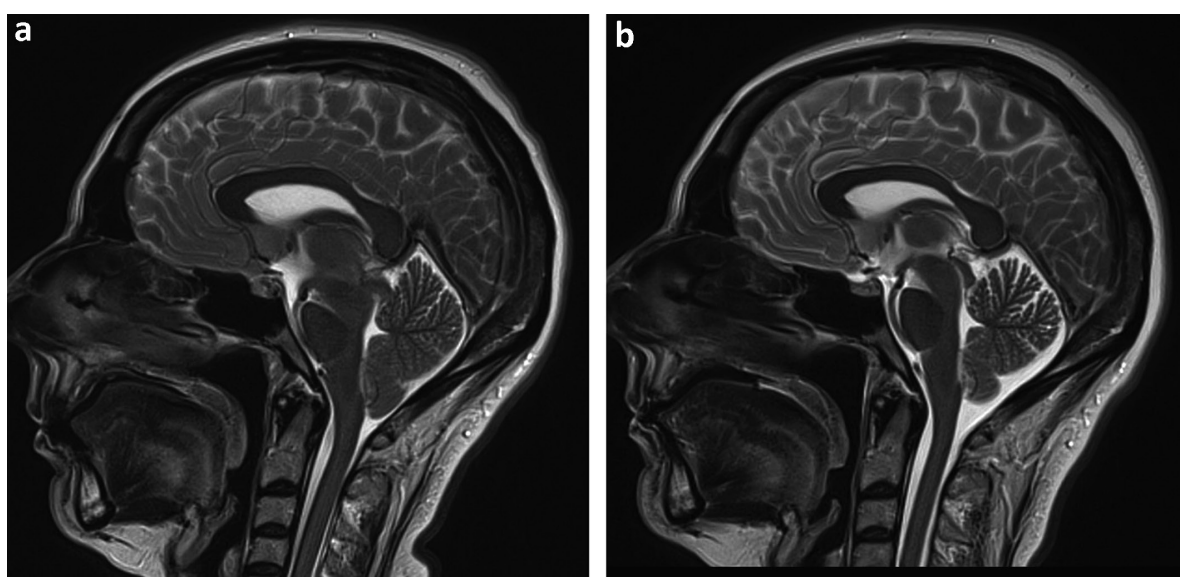
good response (13/23; 57\%). Many of the remaining patients are still undergoing treatment. Only one patient had surgery, which successfully sealed their CSF leak.

A small but significant number of patients who showed a good response to treatment for at least 6 months subsequently went on to relapse, with their symptoms recurring sometime later. This was the case for $10 \%$ of EBP responders and $23 \%$ of targeted treatment responders in our cohort. Relapse rates have not previously been discussed to our knowledge. The majority of these relapsing patients responded to further treatment.

These results are very positive and will hopefully provide evidence that effective treatments for patient with SIH are available, with EBP forming the mainstay of treatment and myelography and targeted treatments providing good results in those patients who do not respond to EBP. Surgery can be reserved for those in whom less invasive interventions do not work. We use these data to inform our discussions with patients, enabling us to give approximate success rates for the various interventions at our institution.

Although most of our SIH patients do fulfil well established clinical and imaging criteria, some do not. We have presented data for our entire cohort to show that they are in practice a complex, heterogeneous group of patients, many of whom undergo extensive investigations and multiple treatments. Treatment responses can be difficult to assess and close follow-up is essential. In our experience, many patients have other headache disorders, migraine in particular, which can pre-date, run concurrently with or develop after SIH, thus complicating the clinical picture. Headache does, of course, have a degree of subjectivity and can be influenced by numerous factors. Indeed the nature of the headache associated with SIH has been shown to vary greatly between patients and can change with leak duration. ${ }^{14}$ Reviewing our data, and appreciating the difficulty of assessing treatment response in many patients, has encouraged us to design a headache diary to assist in patient follow-up.

It should be noted that since we are a regional neurosciences centre with a known interest in this condition, our cohort is likely to be biased towards more refractory cases and those requiring more complex intervention. In addition, our reported relapse rates may be under-estimated as some of these patients have only been treated in the last 12 months and some may go on to relapse at a future date.

Development and delivery of this service has been possible because of the close working relationships between interested consultants from neuroradiology, obstetric anaesthesia, neurology and neurosurgery. As the condition becomes more widely recognised by neurologists and physicians, initial EBP treatment may not need to be delivered in regional neurosciences centres.

In summary, we have presented data for a representative, diverse SIH cohort, and shown the success of a staged, multidisciplinary approach to treatment. We have described our techniques and shown that the use of EBP, with myelography and targeted treatments reserved for those not responding to EBP, produces good results with the majority of patients attaining symptom relief. We have also reported data on relapse rates and response to repeat treatments. Further studies will investigate whether certain imaging or clinical characteristics can be used to predict outcome or relapse, and which type of spinal leak responds best to which intervention.

\section{Conflicts of interest}

Dr David Butteriss is a member of the Medical Advisory Committee for The CSF Leak Association (Scottish charitable incorporated organisation; charity number: SC046319).

\section{References}

1 Schievink WI. Spontaneous spinal cerebrospinal fluid leaks and intracranial hypotension. JAMA 2006;295:2286-96.

2 Kranz PG, Gray L, Malinzak MD, Amrhein T]. Spontaneous intracranial hypotension: pathogenesis, diagnosis, and treatment. Neuroimaging Clin N Am 2019;29:581-94.

3 Schievink WI, Maya MM, Moser F, Tourje J, Torbati S. Frequency of spontaneous intracranial hypotension in the emergency department. J Headache Pain 2007:8:325-8.

4 Schievink WI, Maya MM, Jean-Pierre S et al. A classification system of spontaneous spinal CSF leaks. Neurology 2016;87:673-9.

5 Mokri B. The Monro-Kellie hypothesis. Applications in CSF volume depletion. Historical Neurology 2001;56:1746-8.

6 Mokri B, Posner JB. Spontaneous intracranial hypotension: the broadening clinical and imaging spectrum of CSF leaks. Neurology 2000;55:1771-2.

7 Kranz PG, Malinzak MD, Amrhein TJ, Gray L. Update on the diagnosis and treatment of spontaneous intracranial hypotension. Curr Pain Headache Rep 2017;21:37.

8 Kranz PG, Amrhein T], Choudhury KR, Tanpitukpongse TP, Gray L. Time-dependent changes in dural enhancement associated with spontaneous intracranial hypotension. AJR 2016;207:1283-7.

9 Amoozegar F, Guglielmin D, Hu W, Chan D, Becker W]. Spontaneous intracranial hypotension: Recommendations for management. Can J Neurol Sci 2013;40:144-57.

10 Sencakova D, Mokri B, McClelland RL. The efficacy of epidural blood patch in spontaneous CSF leaks. Neurology 2001;57:1921-3.

11 Wu JW, Hseu SS, Fuh JL et al. Factors predicting response to the first epidural blood patch in spontaneous intracranial hypotension. Brain 2017:140:344-52.

12 Schievink WI, Maya MM, Chu RM, Moser FG. False localizing sign of cervico-thoracic CSF leak in spontaneous intracranial hypotension. Neurology 2015;84:2445-8.

13 Schievink WI, Maya MM, Tourje J. False localizing sign of C1-2 cerebrospinal fluid leak in spontaneous intracranial hypotension. J Neurosurg 2004;100:639-44.

14 Schievink WI, Deline CR. Headache secondary to intracranial hypotension. Curr Pain Headache Rep 2014;18:457.

Address for correspondence: Dr Joanna E Perthen, Department of Neuroradiology, Royal Victoria Infirmary, Queen Victoria Road, Newcastle Upon Tyne NE1 4LP, UK. Email: Joanna.Perthen@nhs.net 